PROCEEDINGS OF THE

AMERICAN MATHEMATICAL SOCIETY

Volume 137, Number 2, February 2009, Pages 431-437

S 0002-9939(08)09531-2

Article electronically published on August 18, 2008

\title{
POLYNOMIAL EXTENSIONS OF IDF-DOMAINS AND OF IDPF-DOMAINS
}

\author{
P. MALCOLMSON AND F. OKOH
}

(Communicated by Bernd Ulrich)

\begin{abstract}
An integral domain is IDF if every non-zero element has only finitely many non-associate irreducible divisors. We investigate when $R$ IDF implies that the ring of polynomials $R[T]$ is IDF. This is true when $R$ is Noetherian and integrally closed, in particular when $R$ is the coordinate ring of a non-singular variety. Some coordinate rings $R$ of singular varieties also give $R[T]$ IDF. Analogous results for the related concept of IDPF are also given. The main result on IDF in this paper states that every countable domain embeds in another countable domain $R$ such that $R$ has no irreducible elements, hence vacuously IDF, and the polynomial ring $R[T]$ is not IDF. This resolves an open question. It is also shown that some subrings $R$ of the ring of Gaussian integers known to be IDPF also have the property that $R[T]$ is not IDPF.
\end{abstract}

\section{INTRODUCTION}

Grams and Warner introduced the concept of IDF (irreducible-divisors-finite) in [8]. This concept has been further studied in [3, 2, and [1]. The following question was raised in 3 and also in 1]: Does $R$ IDF imply that the ring of polynomials $R[T]$ is IDF? We say that IDF ascends to $R[T]$ if this is true. For a non-zero element $a$ in an integral domain, $R$, let $D_{n}(a)$ denote the set of non-associate irreducible divisors of $a^{n}$. The domain $R$ is IDPF (irreducible-divisors-of-powers-finite) if for every non-zero element $a$ in $R$, the union $\bigcup_{n=1}^{\infty} D_{n}(a)$ is finite. The domain is IDF if $D_{1}(a)$ is finite for every non-zero element $a$. The ascent question can also be asked about IDPF and was asked in [12].

We give some examples when IDF and IDPF ascend to $R[T]$. First we recall that a domain is atomic if every non-zero element that is not a unit is a finite product of irreducible elements (atoms) in $R$. Any Noetherian domain is atomic. It was shown in [2] that if $R$ is atomic and IDF, then IDF ascends to $R[T]$. We shall see that $R$ atomic and IDPF need not imply that $R[T]$ is IDPF. The standard proof that factoriality ascends to $R[T]$ factorial adapts to show that if $R$ is GCD and IDF (respectively, IDPF), then IDF (respectively, IDPF) ascends to $R[T]$. The ring of algebraic integers, $\overline{\mathbf{Z}}$, is vacuously IDPF because it has no atoms. Also $\overline{\mathbf{Z}}$ is Bézout, (see Theorem 102 of [10]), hence GCD. Consequently, IDF and IDPF ascend from

Received by the editors October 3, 2005, and, in revised form, January 18, 2008.

2000 Mathematics Subject Classification. Primary 13F20, 13F15; Secondary 13F05, 13B25, $13 \mathrm{G} 05$.

Key words and phrases. Factorization, polynomials, irreducible, IDF, IDPF.

(C)2008 American Mathematical Society Reverts to public domain 28 years from publication 
$\overline{\mathbf{Z}}$ to $\overline{\mathbf{Z}}[T]$. As pointed out in [15, the statement on ascent is also valid if GCD is replaced by pre-Schreier. See [4] for the definition of pre-Schreier.

Theorem 2.2 shows that some subrings of the ring of Gaussian integers provide concrete counterexamples to ascent for IDPF. Counterexamples to ascent for IDF and IDPF follow from Theorem 2.4. The inductive construction of the domain in the proof of this theorem bears some resemblance to Artin's construction of the algebraic closure of a field in [11. The examples in [7, 13, and in Proposition 2.4 of $[12$ are also constructed inductively.

In this paper all rings are integral domains and $U(R)$ will denote the unit group of $R$. Also $R^{\prime}$ will denote the integral closure of $R, R[T]$ denotes the polynomial ring in the variable $T$, and $K$ denotes a field.

We thank Muhammad Zafrullah for his interest in this work and for sending us relevant papers.

\section{EXAMPLeS OF ASCENT}

We begin by recalling several results.

Theorem 1.1 (Finiteness of Integral Closure ([16, Volume I, Chapter V, Theorem 9)). Let $A$ be an integral domain which is a finitely generated algebra over $k$. Let $K$ be the quotient field of $A$ and let $L$ be a finite algebraic extension of $K$. Then the integral closure $A^{\prime}$ of $A$ in $L$ is a finitely generated $A$-module, and also a finitely generated $K$-algebra.

Theorem 1.2 (2], Corollary 3). Let $R$ be a Noetherian domain with $R^{\prime}$ a finitely generated $R$-module. Then $R$ is atomic and IDF if and only if $U\left(R^{\prime}\right) / U(R)$ is finite.

Theorem 1.2 implies that the subrings of the Gaussian integers that contain the ring of integers are all IDF. Proposition 2.16 of [12 tells us when they are also IDPF. Theorem 1.2 also implies that if $R$ is a $K$-subalgebra of $K[T]$ that contains all but finitely many monomials of $K[T]$, then $R$ is IDF.

Theorem 1.3 ([3], Proposition 5.3). A domain $R$ is atomic and IDF if and only if $R[T]$ is atomic and $I D F$.

Theorem 1.4 ([12], Corollary 3.3). A Krull domain, in particular a Noetherian integrally closed domain, is IDPF.

Let $R$ be the coordinate ring of a non-singular variety. The localization of $R$ at each of its maximal ideals is a regular local ring, hence factorial, by Theorem 5.1 of [9]. This implies that $R$ is integrally closed. As $R$ is also Noetherian, $R$ is Krull. Thus $R[T]$ is also Krull (see [6], VII, Section 1, Proposition 13). Thus we get the following corollary of Theorem 1.4 .

Corollary 1.5. The coordinate ring $R$ of a non-singular variety is IDPF and IDPF ascends to $R[T]$.

Theorem 1.1 provides more examples of ascent of IDPF starting with $A$ as the ring $R$ of Corollary 1.5 .

Before continuing let us show that the IDPF-analogue of Theorem 1.2 fails. Let $R$ be a $K$-subalgebra of $K[T]$ that contains all but finitely many monomials of $K[T]$, for example $R=K\left[T^{2}, T^{3}\right]$. In [12] it was shown in Proposition 4.1 that when char $K=0$, then $R$ is not IDPF. (Specifically, $T^{2}(T-1)^{n}, n \geq 1$, is irreducible in 
$K\left[T^{2}, T^{3}\right]$ and $\left(T^{2}-T^{3}\right)^{n}=T^{2}(1-T)^{n} T^{2 n-2}$. Thus, $\mathrm{D}\left(T^{2}(T-1)\right)$ is infinite. Hence $R=K\left[T^{2}, T^{3}\right]$ is not IDPF.) On the other hand, $R^{\prime}=K[T]$ and $U(R)=U(K[T])$.

A simpler example is provided by $\mathbf{Z}[5 i]$, which has unit group of finite index in that of $\mathbf{Z}[i]$ but the former is not IDPF, by Proposition 2.16 of [12].

The failure of the IDPF-analogue of Theorem 1.3 is more involved. It will provide an explicit example of the failure of ascent for IDPF in Section 2 ,

Results and examples in 12 suggest that IDPF may distinguish types of singularities of varieties. We have no proofs, but in dimension $>1$, Example 1.6 shows that the coordinate ring of a singular variety may be IDPF. Coincidentally, within this example is an easy example of an integrally closed local ring that is not regular.

Example 1.6. Let $R=K[X, Y, Z] /\left\langle X Y-Z^{2}\right\rangle$. Let $M=\langle X, Y, Z\rangle \subseteq R$. In $R_{M}$ we have $X Y=Z^{2}$. Thus $R_{M}$ is not factorial, and hence not a regular local ring (see [10, Chapter 4). But $R$ is integrally closed because it is equal to $R\left[X^{-1}\right] \cap R\left[Y^{-1}\right]$ (or see Lemma 2.12 of [13]). Therefore, $R$ is Noetherian and integrally closed, so $R$ is IDPF, by Theorem 1.4. This is also an example of the coordinate ring of a singular variety for which IDPF ascends.

Lest we leave the impression that the coordinate rings of varieties are always IDF, we give the following examples.

Example 1.7. Let $R=K[X, Y] /\left\langle Y^{2}-\left(X^{4}-X^{3}\right)\right\rangle$, where char $K=0$. Since $R$ is Noetherian, it is atomic. The equation $\left(\frac{Y}{X}\right)^{2}=X^{2}-X$ shows that $R^{\prime}$ contains $\frac{Y}{X}$.

Let $p=2 \frac{Y}{X}+2 X-1$. Then $p^{-1}=-2 \frac{Y}{X}+2 X-1$ and $p$ are in $R^{\prime}$. Therefore, $R^{\prime}=K\left[p, p^{-1}\right]$ and $U\left(R^{\prime}\right) / U(R)$ is infinite, so by Theorem [1.2, $R$ is not IDF.

Example 1.8. Again in characteristic zero, consider the coordinate ring of the curve $X^{2}\left(1+Y^{2}\right)^{3}=\left(1-Y^{2}\right)^{2}$. We have the unit $\left(1+Y^{2}\right)^{-1}=\frac{1}{4}\left[\left(1+Y^{2}\right)^{2} X^{2}+\right.$ $3-Y^{2}$ ]. In $R^{\prime}$, we have $p=\frac{\left(1+Y^{2}\right)^{2} X}{1-Y^{2}}$ with $p^{2}=1+Y^{2}$, a unit. Also in $R^{\prime}$, we have $q=p+Y$ and $q^{-1}=p-Y$. Here $2 p q=\left(q+q^{-1}\right) q=q^{2}+1$, a unit. No power of $q^{2}+1$ is in $R$ since none is uniquely defined at the point $(X=0, Y=1)$ of the curve. So $R^{\prime}=K\left[q, q^{-1},\left(q^{2}+1\right)^{-1}\right]$. As in Example 1.7 $R$ is not IDF.

The next result is an IDF/IDPF analogue of the classical result on factorial domains. Our proof is an adaptation of the proof in Chapter 4 of [11]. We refer to [10. pp. 32-42], for properties of GCD-domains. In particular, if $R$ is GCD, so is $R[T]$.

Theorem 1.9. If $R$ is a GCD-domain and IDF (respectively, IDPF), then $R[T]$ is also $G C D$ and IDF (respectively, IDPF).

Proof. The classical argument using content shows that the multiplicative set of primitive polynomials in $R[T]$ has unique factorization into irreducibles - the same irreducibles as in $K[T]$, where $K$ is the quotient field of $R$. (This implies that every irreducible polynomial in $R[T]$ is prime.)

We now show that $R[T]$ is also IDF (respectively, IDPF). Let $f \in R[T]$. Since $R$ is IDF (respectively, IDPF), we may assume that $f$ has positive degree. Since $R$ is GCD, we may assume that $f$ is primitive.

Since irreducibles are prime, if $f=p_{1} \cdots p_{r}$ where $p_{i}$ is irreducible, then these $p_{i}$ are the only irreducibles dividing $f$. We deduce that there are at most $\operatorname{deg} f$ of them. For the same reason, $\mathrm{D}(f)=\mathrm{D}_{1}(f)$, so $R[T]$ is IDF (respectively, IDPF). 
It follows from Theorem 1.9 that if $R$ is a valuation domain, then $R[T]$ is IDPF, hence IDF, a fact already noted for IDF in $[3]$.

In 12] we determined when subrings of the Gaussian integers are IDPF. In some of these cases IDPF ascends to the polynomial ring. This allows us to give an example of ascent which is not integrally closed.

Theorem 1.10. If $n=2^{k}$ for some positive integer $k$, then $\mathbf{Z}[n i][T]$ is IDPF.

Proof. We first prove that if $f \in \mathbf{Z}[i][T]$, then $f^{n} \in \mathbf{Z}[n i][T]$. Write $f=A+B i$, where $A$ and $B$ are polynomials in $\mathbf{Z}[T]$. As shown in Lemma 2.11 of $\left[12, \frac{2^{k}}{2 l+1}\right.$ is divisible by $2^{k}$ for $l$ a positive integer and $2 l+1<2^{k}$. These are the imaginary terms in the binomial expansion of $(A+B i)^{n}$. Therefore, $(A+B i)^{n} \in \mathbf{Z}[n i][T]$.

We now imitate the proof of Proposition 2.13 of $\left[12\right.$ with $n=2^{k}$. We have the analogue of Lemma 2.12 of [12] with $\mathbf{Z}[i][T]$ and $\mathbf{Z}[n i][T]$ replacing $\mathbf{Z}[i]$ and $\mathbf{Z}[n i]$ respectively: for $f \in \mathbf{Z}[n i][T]$, if $D(f)$ is infinite, then there is a factor $f_{1}$ of $f$ in $\mathbf{Z}[i][T]$ such that for any integer $l>0$, there is an integer $j$ with $j n>l$ and $f_{1}^{j n}$ in $\mathbf{Z}[n i][T]$. Write $f_{1}^{n}=E+n F i$ for $E, F$ polynomials in $\mathbf{Z}[T]$.

Suppose now that the content of $E$ is odd. Choose $g \in D(f)$ so that the exponent on $f_{1}$ is greater than $2 n$. Then $g=f_{1}^{2 n} g_{1}=(E+n F i)^{2} g_{1}$ for some $g_{1} \in \mathbf{Z}[i][T]$. Examining imaginary parts and recalling that $g \in \mathbf{Z}[n i][T]$ gives that $n \mid E^{2} \operatorname{Im} g_{1}$. Since the content of $E^{2}$ is odd, $n \mid \operatorname{Im} g_{1}$. Thus $g_{1} \in \mathbf{Z}[n i][T]$. This contradicts the irreducibility of $g$.

Finally, suppose $2^{m}$ is the largest power of 2 that divides the content of $E$. (In the case treated above, $m=0$.) Then $n$ divides $E^{l}$ for some positive integer $l$. Choose $g \in D(f)$ so that the exponent on $f_{1}$ is greater than $2 l n$. Then $g=$ $f_{1}^{2 l n} g_{1}=(E+n F i)^{2 l} g_{1}$. As above, $n$ divides $E^{l} \operatorname{Im} g_{1}$, so $(E+n F i)^{l} g_{1} \in \mathbf{Z}[n i][T]$. But now $g$ has a factor of $(E+n F i)^{l}$ in $\mathbf{Z}[n i][T]$, contradicting that $g$ is irreducible. Hence $D(f)$ is finite for every $f \in \mathbf{Z}[n i][T]$, so $\mathbf{Z}[n i][T]$ is IDPF.

\section{Counterexamples to Ascent}

The first result in this section provides a family of explicit counterexamples to ascent of IDPF from $R$ to $R[T]$.

In [12] it is shown that if the positive integer $n$ has no prime divisors $p$ with $p \equiv 1(\bmod 4)$, then the subring $R=\mathbf{Z}[n i]=\{a+n b i: a, b \in \mathbf{Z}\}$ of the Gaussian integers is IDPF. Since $R$ is Noetherian, it is atomic and by Theorem 1.2 it is IDF. Hence by Theorem $1.3, R[T]$ is IDF. Theorem 2.2 shows that $R[T]$ is not IDPF.

Lemma 2.1. Let $k$ be an odd positive integer. For any odd prime $p$ that divides $k$, let $k=p^{r} m$, where $p$ does not divide $m$. Then $p^{r}$ does not divide $\frac{p^{r} m}{p^{r}}$.

Proof. We need only consider the factors of $\frac{p^{r} m}{p^{r}}$ that are divisible by $p$. The latter is $\frac{p^{r} m\left(p^{r} m-p\right) \cdots\left(p^{r} m-\left(p^{r}-p\right)\right)}{p^{r}\left(p^{r}-p\right) \cdots\left(p^{r}-\left(p^{r}-p\right)\right)}$. Comparing the $p$-factors of the numerator and denominator and noting that $p$ does not divide $m$, we see that $p$ does not divide $\frac{p^{r} m}{p^{r}}$. Hence $p^{r}$ does not divide $\frac{p^{r} m}{p^{r}}$.

Theorem 2.2. Let $n$ be a positive integer for which $\mathbf{Z}[n i]$ is IDPF. If $n$ is not a power of 2 , then $\mathbf{Z}[n i][T]$ is not IDPF.

Proof. We first show that for $k$ any odd positive integer, the polynomial $f_{n}=$ $n(1+2 i T)^{k}$ is irreducible in $\mathbf{Z}[n i]$ or has a factor of the form $n_{1}(1+2 i T)^{k}$, where 
$n_{1} \mid n$. Since $(1+2 i T)$ is irreducible in $\mathbf{Z}[i][T]$, any factorization of $n(1+2 i T)^{k}$ is of the form $\left(n_{1}(1+2 i T)^{k_{1}}\right)\left(n_{2}(1+2 i T)^{k_{2}}\right)$, where one of $k_{1}$ and $k_{2}$ is odd and the other is even. Suppose $k_{1}$ is odd and $1<n_{1}<n$. In order for a polynomial to be in $\mathbf{Z}[n i][T]$, the coefficient of each monomial with imaginary coefficient must be a multiple of $n$. Therefore $n \mid 2^{k_{1}} n_{1}$. (This is the coefficient of the last term of the binomial expansion of $n_{1}(1+2 i T)^{k_{1}}$. It is imaginary because $k_{1}$ is odd.) With $n=2^{l} s$ with $s$ an odd integer, we get that $s \mid n_{1}$. So $n_{1}=s s_{1}$ for some positive integer $s_{1}$. Now go to $k_{2}$. Then $n \mid\left(\begin{array}{c}2 n_{2} k_{2} \\ 1\end{array}\right)$. (This is the second term in the binomial expansion of $n_{2}(1+2 i T)^{k_{2}}$ and is imaginary.) Suppose some odd divisor, $s_{2}>1$, of $n$ divides $n_{2}$. This contradicts the fact that the odd component, $s$, of $n$ divides $n_{1}$ and $n=n_{1} n_{2}$. Therefore, some odd prime divisor of $n$ divides $k_{2}$. Lemma 2.1 implies that some imaginary component of $n_{2}(1+2 i T)^{k_{2}}$ is not divisible by $n$. So in the alleged factorization of $n(1+2 i T)^{k}$, some factor is not in $\mathbf{Z}[n i][T]$. If $k_{1}=k$, the above argument gives $n_{1} \mid n$. So we have proved that for $k$ any odd positive integer the polynomial $f_{n}=n(1+2 i T)^{k}$ is irreducible in $\mathbf{Z}[n i]$ or has a factor of the form $n_{1}(1+2 i T)^{k}$, where $n_{1} \mid n$.

One of these irreducible factors $n_{1}(1+2 i T)^{k}$ divides $(n(1+2 i T))^{k}$ so that $D(n(1+2 i T))$ is infinite. So if $n$ is not a power of 2 , then $\mathbf{Z}[n i][T]$ is not IDPF.

To construct a counterexample for ascent of IDF, first we describe a general construction. Let $S$ be any domain and $r \in S$ a non-zero non-unit. Consider $\sigma_{r}(S)=S[X, Y] /\langle X Y-r\rangle$. Then we have shown in [12] that $\sigma_{r}(S)$ is a domain with no new units. We can also deduce this from the lemma below using $t=0,1$.

Lemma 2.3. If $t \in S$ and $f \in \sigma_{r}(S)$ is a divisor of $t$ in $\sigma_{r}(S)$, then $f$ is of the form $a X^{n}$ or $a Y^{n}$ with $a \in S$, and ar ${ }^{n}$ is a divisor of $t$ in $S$.

Proof. Let $f g=t$ for $f, g \in \sigma_{r}(S)$ and let $f=a_{-n} Y^{n}+\cdots+a_{-1} Y+a_{0}+a_{1} X+$ $\cdots+a_{m} X^{m}, g=b_{-k} Y^{k}+\cdots+b_{l} X^{l}$. As in ([12]) this implies $f=a_{n} X^{n}$ and $g=b_{n} Y^{n}$ (or vice versa). Thus $f g=t=a_{n} b_{n} r^{n}$.

We refer to $\sigma_{r}(S)$ as a splitting of $r$ in $S$, since it provides new factors without new units. Note that $\sigma$ can be iterated (with new polynomial variables) to split many elements. In particular, $\sigma_{r}^{\infty}$ splits $r$ in infinitely many ways.

Lemma 2.4. Let $D$ be a domain and let $R=D\left[A_{i}, B_{i}, C_{i}, i=1,2, \ldots\right] / I$, where $I$ is the ideal of the polynomial ring generated by

$$
\begin{aligned}
& p_{i}=A_{i} C_{i}-A_{1} C_{1} \text { for } i>1, \\
& q_{i}=B_{i} C_{i}-B_{1} C_{1} \text { for } i>1, \\
& r_{i j}=A_{i} B_{j}-A_{j} B_{i} \text { for } i<j .
\end{aligned}
$$

Order the variables by $A_{1} \ll B_{1} \ll C_{1} \ll A_{2} \ll B_{2} \ll \ldots$ (so that $A_{1}^{n}<B_{1}$ for any $n$, etc.). Then the relations $p_{i}, q_{i}, r_{i j}$ form a Gröbner basis for $I$ over $D$. Thus $R$ can be regarded as the reduced polynomials with respect to these relations.

Proof. The corresponding $S$-polynomials (as in [14]) are as follows:

$$
S\left(p_{i}, p_{j}\right)= \begin{cases}A_{1} C_{1} A_{j} C_{j}-A_{1} C_{1} A_{i} C_{i} & \text { if } i \neq j, \\ 0 & \text { if } i=j .\end{cases}
$$

The former reduces using $p_{i}$ and $p_{j}$. Also

$$
S\left(p_{i}, r_{j k}\right)= \begin{cases}A_{i} C_{i} A_{k} B_{j}-A_{1} C_{1} A_{j} B_{k} & \text { if } i \neq j(<k), \\ A_{k} B_{i} C_{i}-A_{1} C_{1} B_{k} & \text { if } i=j(<k) .\end{cases}
$$


The former reduces using $p_{i}$ and $r_{j k}$. The latter reduces using $r_{i k}$ and $q_{i}$.

Similarly for the $q_{j}$ 's,

$$
S\left(p_{i}, q_{j}\right)= \begin{cases}A_{i} C_{i} B_{1} C_{1}-B_{j} C_{j} A_{1} C_{1} & \text { if } i \neq j, \\ A_{i} B_{1} C_{1}-A_{1} C_{1} B_{i} & \text { if } i=j .\end{cases}
$$

The former reduces using $p_{i}$ and $q_{j}$. The latter reduces using $r_{1 i}$. Also

$$
S\left(r_{i j}, r_{k l}\right)= \begin{cases}A_{i} B_{j} A_{l} B_{k}-A_{j} B_{i} A_{k} B_{l} & \text { if } i \neq k, j \neq l, \\ A_{l} B_{i} B_{j}-A_{j} B_{1} B_{l} & \text { if } i=k, j \neq l, \\ A_{l} A_{l} B_{k}-A_{l} A_{k} B_{i} & \text { if } i \neq k, j=l, \\ 0 & \text { if } i=k, j=l .\end{cases}
$$

The first reduces using $r_{i j}$ and $r_{k l}$. The second reduces using $r_{j l}$ or $r_{l j}$. The third reduces using $r_{i k}$ or $r_{k i}$.

Since all $S$-polynomials reduce to 0 with respect to the relations, the relations form a Gröbner basis of the ideal $I$ (see [14, Theorem 1.2.1). In fact this is a reduced Gröbner basis of $I$.

Alternatively, we may check the overlap ambiguities of Bergman ( 5 ).

An overlap ambiguity occurs in $A_{j} B_{j} C_{j}$ for $j>1$. But $A_{j} B_{j} C_{j}$ reduces to $A_{1} B_{j} C_{1}$, which in turn reduces to $A_{j} B_{1} C_{1}$. The other way, $A_{j} B_{j} C_{j}$ reduces immediately to $A_{j} B_{1} C_{1}$.

Similarly, for $j>i>1, A_{i} B_{j} C_{j}$ reduces to $A_{j} B_{i} C_{j}$, and then to $A_{1} B_{i} C_{1}$. Also $A_{i} B_{j} C_{j}$ may reduce to $A_{i} B_{1} C_{1}$, and thence to $A_{1} B_{i} C_{1}$.

Recall from 3 that an irreducible element is also called an atom. Consequently we say that a domain with no atoms is atomless.

Theorem 2.5. Every countable integral domain embeds in a countable atomless domain $R_{\infty}$ such that $R_{\infty}[T]$ is not IDF.

Proof. Let $D$ be any countable domain and construct the $R$ of Lemma 2.4 Since the relations form a Gröbner basis we regard $R$ as the reduced elements of the polynomials in the $A, B, C$.

Note that there are no reductions involving $B_{1}$. Hence if $B_{1} f=0$, and $f$ is reduced, then there are no reductions in $B_{1} f$, so $f=0$ already. So $B_{1}$ is a non-zero divisor in $R$. Thus $R$ embeds in $R\left[B_{1}^{-1}\right]$. But $R\left[B_{1}^{-1}\right] \cong \sigma_{B_{1} C_{1}}^{\infty}\left(D\left[A_{1}, B_{1}, B_{1}^{-1}, C_{1}\right]\right)$ with the $B_{i}$ and $C_{i}$ providing the splittings for $B_{1} C_{1}$ and $A_{j}=A_{1} B_{1}^{-1} B_{j}$. This is a domain by the remark preceding Lemma 2.3. So $R$ is a domain.

Now note that $R$ is graded (with each variable of degree one). Thus the only units of $R$ are those of $D$, and there are no linear relations (the grade one part of $R$ is free over $D$ on the variables). Thus each variable is irreducible.

Now $R$ is countable, so let $R_{1}$ be the union of the splittings $\sigma_{r}(R)$ for each of the countably many irreducibles $r$ in $R$ (up to associates). Repeat the procedure to get $R_{\infty}=\bigcup R_{i}$, having no irreducibles, or enumerate the irreducibles $\left\{r_{i}\right\}$ of $R$ and form $R_{1}=\ldots \sigma_{r_{3}}\left(\sigma_{r_{2}}\left(\sigma_{r_{1}}(R)\right)\right)$, still countable. Repeat to get $R_{2}, R_{3}$, etc., and $R_{\infty}=\bigcup R_{i}$.

Suppose $A_{i}, B_{i}$ have a common non-unit divisor $f$ in $R_{\infty}$. Let $f \in R_{k}$ where $k$ is minimal. Now $R_{k-1}=R_{k-1}^{(0)} \subseteq R_{k-1}^{(1)} \subseteq \ldots \subseteq R_{k}$, where each $R_{k-1}^{(j)}=\sigma_{r_{j}}\left(R_{k-1}^{(j-1)}\right)$ for an irreducible $r_{j} \in R_{k-1}$. So assume $f \in \sigma_{r_{j}}\left(R_{k-1}^{(j-1)}\right)$ for $j$ minimal. By the lemma, $f=a X^{n}$ (or $\left.a Y^{n}\right)$ ) with $a \in R_{k-1}^{(j-1)}$ and $A_{i}$ and $B_{i}$ have the common nonunit divisor $a \in R_{k-1}^{(j-1)}$. Since $j$ was minimal, $A_{i}$ and $B_{i}$ have a common non-unit 
divisor in $R_{k-1}$. Since $k$ was minimal, $A_{i}$ and $B_{i}$ have a common non-unit factor in $R$. Both are irreducible in $R$, hence associated. Since $R$ is graded, any unit of $R$ is in $D$. But $\alpha A_{i}$ cannot be reduced to $B_{i}$ for any $0 \neq \alpha \in K$. Hence we have a contradiction, and so $A_{i}$ and $B_{i}$ have no common factor in $R_{\infty}$.

Now let $A_{i} C_{i}=A$ and $B_{i} C_{i}=B$. In $R_{\infty}[T]$, the polynomial $A T+B$ has infinitely many non-associated irreducible divisors $A_{i} T+B_{i}$ for $i=1,2, \ldots$. Therefore, $R_{\infty}[T]$ is not IDF, while $R_{\infty}$ has no irreducibles, so it is vacuously IDF.

Some problems.

(1) It is likely that countable is dispensable in Theorem 2.5. but we do not know.

(2) In 12 there are various examples of varieties whose coordinate rings are or are not IDPF. One could then ask for a geometric characterization of those varieties whose coordinate rings are IDPF.

\section{REFERENCES}

1. D. F. Anderson and D. N. El Abidine, Factorization in integral domains. III, J. Pure Appl. Algebra 135 (1999), 107-127. MR 1667552 (2000k:13020)

2. D. D. Anderson and B. Mullins, Finite factorization domains, Proc. Amer. Math. Soc. 124 (1996), 389-396. MR1322910|(96i:13001)

3. D. D. Anderson, D. F. Anderson, and M. Zafrullah, Factorization in integral domains, J. Pure Appl. Algebra 69 (1990), 1-19. MR.1082441 (92b:13028)

4. D. D. Anderson and M. Zafrullah, The Schreier property and Gauss's lemma, Boll. Unione Mat. Itali. Sez. B Artic. Ric. Mat. (8) 10 (2007), 43-62. MR2310957 (2008c:13001)

5. G. M. Bergman, The diamond lemma for ring theory, Adv. Math. 29 (1978), 178-218. MR.506890 (81b:16001)

6. N. Bourbaki, Commutative algebra, Elements of Mathematics, Chapters 8 and 9, SpringerVerlag, 1989. MR2284892 (2007h:13001)

7. J. Coykendall and M. Zafrullah, AP-domains and unique factorization, J. Pure Appl. Algebra 189 (2004), 27-35. MR 2038561 (2004j:13029)

8. A. Grams and H. Warner, Irreducible divisors in domains of finite character, Duke Math. J. 42 (1975), 271-284. MR0376661 (51:12836)

9. R. Hartshorne, Algebraic Geometry, Springer-Verlag, 1977. MR0463157 (57:3116)

10. I. Kaplansky, Commutative Rings, Polygonal Publishing House, Passaic, New Jersey, 1994.

11. S. Lang, Algebra, Third Edition, Addison Wesley, 1993. MR0197234 (33:5416)

12. P. Malcolmson and F. Okoh, A class of integral domains between factorial domains and IDFdomains, Houston J. Math. 32 (2006), no. 2, 399-421. MR2219322 (2007b:13034)

13. M. Roitman, Polynomial extensions of atomic domains, J. Pure Appl. Algebra 87 (1993), 187-199. MR1224218 (94e:13042)

14. W. V. Vasconcelos, Computational methods in commutative algebra and algebraic geometry, Springer, 1998. MR 1484973(99c:13048)

15. M. Zafrullah, Email communication (2005).

16. O. Zariski and P. Samuel, Commutative Algebra, Von Nostrand, Princeton, 1960. MR0120249 $(22: 11006)$

Department of Mathematics, Wayne State University, Detroit, Michigan 48202

E-mail address: petem@math.wayne.edu

Department of Mathematics, Wayne State University, Detroit, Michigan 48202

E-mail address: okoh@math.wayne.edu 